\title{
ASYMPTOTIC PERIODICITY OF GRADE ASSOCIATED TO MULTIGRADED MODULES
}

\author{
FUTOSHI HAYASAKA
}

(Communicated by Irena Peeva)

\begin{abstract}
Let $R$ be a Noetherian $\mathbb{N}^{r}$-graded ring generated in degrees $\mathbf{d}_{1}, \ldots, \mathbf{d}_{r}$ which are linearly independent vectors over $\mathbb{R}$, and let $\mathfrak{a}$ be an ideal in $R_{\mathbf{0}}$. In this paper, we investigate the asymptotic behavior of the grade of the ideal $\mathfrak{a}$ on the homogeneous components $M_{\mathbf{n}}$ of a finitely generated $\mathbb{Z}^{r}$-graded $R$-module $M$ and show that the periodicity occurs in a cone.
\end{abstract}

\section{INTRODUCTION}

Let $A$ be a commutative Noetherian ring and $\mathfrak{a}$ an ideal in $A$. Let $R$ be a Noetherian $\mathbb{N}^{r}$-graded ring with $R_{0}=A$, and let $M$ be a finitely generated $\mathbb{Z}^{r}$ graded $R$-module. In this paper, we study the asymptotic behavior of the numerical function grade $\left(\mathfrak{a}, M_{\mathbf{n}}\right)$ associated to $M$.

The first result in this setting is due to McAdam and Eakin [7. They proved that if $R$ is a standard $\mathbb{N}$-graded ring, then the set of primes $\operatorname{Ass}_{A} M_{n}$ is stable for all large $n$, and hence, by using the technique due to Brodmann [3, we have that $\operatorname{grade}\left(\mathfrak{a}, M_{n}\right)$ is constant for all large $n$. They also showed that Brodmann's result [2] about the asymptotic prime divisors of an ideal followed from their results as a direct consequence. Afterwards, a number of authors have extended these results to multigraded cases, especially in connection with the study of the asymptotic prime divisors and the analytic spread of ideals. In particular, West [8] extended McAdam-Eakin's results to multigraded cases. He proved that if $R$ is a standard $\mathbb{N}^{r}$-graded ring, then the set of primes $\operatorname{Ass}_{A} M_{\mathbf{n}}$ is eventually stable, and hence $\operatorname{grade}\left(\mathfrak{a}, M_{\mathbf{n}}\right)$ is constant for all large $\mathbf{n}$. He also considered several interesting nonstandard multigraded cases. For more general results in the standard graded cases, see [1, 5].

On the other hand, when $R$ is a standard $\mathbb{N}$-graded ring with a local ring $A$, Herzog and Hibi [6] gave a direct proof of the stability of $\operatorname{depth}_{A} M_{n}$ by using the Hilbert polynomial of Koszul homology modules of $M$ with respect to the maximal ideal of $A$ instead of the asymptotic stability of $\operatorname{Ass}_{A} M_{n}$. More recently, ColoméNin and Elias [4] investigated in a similar way the asymptotic behavior of the depth associated to graded modules over certain nonstandard multigraded rings.

Received by the editors February 22, 2011.

2010 Mathematics Subject Classification. Primary 13A02; Secondary 13C15.

Key words and phrases. Module, multigraded, grade, cone. 
The purpose of this paper is to give a common generalization to all of the results concerning the asymptotic grade stated above with a more direct approach. The result is the following:

Theorem 1.1. Let $R$ be a Noetherian $\mathbb{N}^{r}$-graded ring generated in degrees $\mathbf{d}_{1}, \mathbf{d}_{2}, \ldots, \mathbf{d}_{r}$, where $\mathbf{d}_{1}, \mathbf{d}_{2}, \ldots, \mathbf{d}_{r} \in \mathbb{N}^{r}$ are linearly independent vectors over $\mathbb{R}$, with $R_{\mathbf{0}}=A$. Let $M=\bigoplus_{\mathbf{n} \in \mathbb{Z}^{r}} M_{\mathbf{n}}$ be a finitely generated $\mathbb{Z}^{r}$-graded $R$-module. Then, for any ideal $\mathfrak{a}$ in $A$, there exists a vector $\mathbf{k} \in \mathbb{N}^{r}$ such that, in the cone $C_{\mathbf{k}}$ with vertex $\mathbf{k}$ generated by $\mathbf{d}_{1}, \mathbf{d}_{2}, \ldots, \mathbf{d}_{r}$, grade $\left(\mathfrak{a}, M_{\mathbf{n}}\right)$ is periodic with respect to $\mathbf{d}_{1}, \mathbf{d}_{2}, \ldots, \mathbf{d}_{r}$. Namely, the equality

$$
\operatorname{grade}\left(\mathfrak{a}, M_{\mathbf{n}}\right)=\operatorname{grade}\left(\mathfrak{a}, M_{\mathbf{n}+\mathbf{m}}\right)
$$

holds true for all $\mathbf{n} \in C_{\mathbf{k}}$ and all $\mathbf{m} \in \Gamma$, where $\Gamma$ is the semigroup generated by $\mathbf{d}_{1}, \mathbf{d}_{2}, \ldots, \mathbf{d}_{r}$.

In the next section, we fix our notation and give some facts about cones and graded modules. In section 3 , we will give a proof of Theorem 1.1.

Throughout this paper, $A$ is a commutative Noetherian ring with identity. $\mathbb{N}$ $($ resp. $\mathbb{R})$ denotes the set of nonnegative integers (resp. real numbers), and $r$ is any fixed positive integer. Vectors will always be written in bold-faced letters, e.g., a, and they will be represented by row vectors, e.g., $\mathbf{a}=\left(a_{1}, a_{2}, \ldots, a_{r}\right)$.

\section{Preliminaries}

Let $\mathbf{d}_{1}, \mathbf{d}_{2}, \ldots, \mathbf{d}_{r} \in \mathbb{N}^{r}$ be any fixed linearly independent vectors over $\mathbb{R}$. We denote by $\Gamma \subseteq \mathbb{N}^{r}$ the semigroup generated by $\mathbf{d}_{1}, \mathbf{d}_{2}, \ldots, \mathbf{d}_{r}$, i.e.,

$$
\Gamma=\left\{\sum_{i=1}^{r} c_{i} \mathbf{d}_{i} \mid c_{i} \in \mathbb{N}\right\} .
$$

For any vector $\mathbf{k} \in \mathbb{N}^{r}$, let

$$
C_{\mathbf{k}}:=\left\{\mathbf{k}+\sum_{i=1}^{r} c_{i} \mathbf{d}_{i} \mid c_{i} \in \mathbb{R}_{\geq 0}\right\} \cap \mathbb{N}^{r}
$$

be the cone with vertex $\mathbf{k}$ generated by $\mathbf{d}_{1}, \mathbf{d}_{2}, \ldots, \mathbf{d}_{r}$ and

$$
\Delta_{\mathbf{k}}:=\left\{\mathbf{k}+\sum_{i=1}^{r} c_{i} \mathbf{d}_{i} \mid 0 \leq c_{i}<1, c_{i} \in \mathbb{R}\right\} \cap \mathbb{N}^{r}
$$

be the basic cell of the cone $C_{\mathbf{k}}$. Then it is easy to see that

(i) $\Delta_{\mathbf{k}}$ is a finite subset of $C_{\mathbf{k}}$,

(ii) for any $\mathbf{n} \in C_{\mathbf{k}}$, there is a unique expression $\mathbf{n}=\delta+\mathbf{m}$ with $\delta \in \Delta_{\mathbf{k}}$ and $\mathbf{m} \in \Gamma$, and

(iii) $C_{\mathbf{k}}=\bigcup_{\delta \in \Delta_{\mathbf{k}}}(\delta+\Gamma)$.

Moreover, we have the following:

Lemma 2.1. For any vectors $\mathbf{k}, \mathbf{k}^{\prime} \in \mathbb{N}^{r}$, there exists an integer $\ell_{0} \geq 0$ such that

$$
\mathbf{k}^{\prime}+\ell\left(\mathbf{d}_{1}+\mathbf{d}_{2}+\cdots+\mathbf{d}_{r}\right) \in C_{\mathbf{k}} \text { for all } \ell \geq \ell_{0} .
$$

In particular, $C_{\mathbf{k}} \cap C_{\mathbf{k}^{\prime}} \neq \emptyset$, and hence there exists a cone $C_{\mathbf{k}^{\prime \prime}}$ such that $C_{\mathbf{k}^{\prime \prime}} \subseteq$ $C_{\mathbf{k}} \cap C_{\mathbf{k}^{\prime}}$. 
Proof. Consider the system

$$
\mathbf{x} D=\mathbf{k}-\mathbf{k}^{\prime}
$$

where $D$ is a square matrix of size $r$ whose $i$-th row is $\mathbf{d}_{i}$. Since $\mathbf{d}_{1}, \mathbf{d}_{2}, \ldots, \mathbf{d}_{r}$ are linearly independent, there is a unique solution $\mathbf{a}=\left(a_{1}, a_{2}, \ldots, a_{r}\right) \in \mathbb{R}^{r}$ of the system (11). Let

$$
\ell_{0}:=\max \left\{\left\lceil\left|a_{i}\right|\right\rceil \mid i=1,2, \ldots, r\right\},
$$

where $\lceil *\rceil$ denotes the least integer $\geq *$. Take $\ell \geq \ell_{0}$ and put $c_{i}:=\ell-a_{i} \in \mathbb{R}_{\geq 0}$. Then $\mathbf{a}=\left(\ell-c_{1}, \ell-c_{2}, \ldots, \ell-c_{r}\right)$ and we have

$$
\mathbf{k}^{\prime}+\ell\left(\mathbf{d}_{1}+\mathbf{d}_{2}+\cdots+\mathbf{d}_{r}\right)=\mathbf{k}+c_{1} \mathbf{d}_{1}+c_{2} \mathbf{d}_{2}+\cdots+c_{r} \mathbf{d}_{r} \in C_{\mathbf{k}} .
$$

The last assertions follow from the fact that the above vector is in $C_{\mathbf{k}^{\prime}}$.

Let $R=\bigoplus_{\mathbf{n} \in \mathbb{N}^{r}} R_{\mathbf{n}}$ be a Noetherian $\mathbb{N}^{r}$-graded ring with $R_{\mathbf{0}}=A$. Assume that $R$ is generated in degrees $\mathbf{d}_{1}, \mathbf{d}_{2}, \ldots, \mathbf{d}_{r}$, i.e., $R=A\left[R_{\mathbf{d}_{1}}, R_{\mathbf{d}_{2}}, \ldots, R_{\mathbf{d}_{r}}\right]$. Let $R_{++}=\left(R_{\mathbf{d}_{1}} R_{\mathbf{d}_{2}} \cdots R_{\mathbf{d}_{r}}\right) R$ be the irrelevant ideal of $R$, which is the ideal generated by homogeneous components of degree $\mathbf{d}_{1}+\mathbf{d}_{2}+\cdots+\mathbf{d}_{r}$. For a finitely generated $\mathbb{Z}^{r}$-graded $R$-module $M=\bigoplus_{\mathbf{n} \in \mathbb{Z}^{r}} M_{\mathbf{n}}$, we define the homogeneous support of $M$ as

$\operatorname{Supp}_{++} M:=\left\{P \in \operatorname{Spec} R \mid P\right.$ is a graded ideal, $M_{P} \neq(0)$, and $\left.R_{++} \nsubseteq P\right\}$.

For any vector $\delta \in \mathbb{N}^{r}$, we set $M^{(\delta+\Gamma)}=\bigoplus_{\mathbf{m} \in \Gamma} M_{\delta+\mathbf{m}}$, which is a graded submodule of $M$.

Lemma 2.2. Let $M=\bigoplus_{\mathbf{n} \in \mathbb{Z}^{r}} M_{\mathbf{n}}$ be a finitely generated $\mathbb{Z}^{r}$-graded $R$-module. Then the following statements are equivalent:

(1) $\operatorname{Supp}_{++} M=\emptyset$;

(2) there exists a vector $\mathbf{k} \in \mathbb{N}^{r}$ such that $M_{\mathbf{n}}=(0)$ for all $\mathbf{n} \in C_{\mathbf{k}}$.

Proof. Suppose $\operatorname{Supp}_{++} M=\emptyset$. Let $\mathbf{k}_{0} \in \mathbb{N}^{r}$ be any fixed vector. Then we claim the following:

Claim. For any $\delta \in \Delta_{\mathbf{k}_{0}}$, there exists $\mathbf{k}=\mathbf{k}(\delta) \in \mathbb{N}^{r}$ such that $M_{\mathbf{n}}=(0)$ for all $\mathbf{n} \in C_{\mathbf{k}} \cap(\delta+\Gamma)$.

Let $\delta \in \Delta_{\mathbf{k}_{0}}$. The assertion is clear if $M^{(\delta+\Gamma)}=(0)$. Assume $M^{(\delta+\Gamma)} \neq(0)$ and write $M^{(\delta+\Gamma)}=R m_{1}+R m_{2}+\cdots+R m_{t}$, where $m_{i} \in M_{\mathbf{k}_{i}}$ and $\mathbf{k}_{i} \in \delta+\Gamma$. Since Supp $_{++} M=\emptyset, \operatorname{Supp}_{++} M^{(\delta+\Gamma)}=\emptyset$ so that $R_{++} \subseteq \sqrt{\operatorname{Ann}_{R}\left(M^{(\delta+\Gamma)}\right)}$. Therefore there exists an integer $\ell \geq 0$ such that $R_{++}^{\ell} \cdot m_{i}=(0)$ for all $i=1,2, \ldots, t$. This implies that

$$
\left[R m_{i}\right]_{\mathbf{k}_{i}+\ell\left(\mathbf{d}_{1}+\mathbf{d}_{2}+\cdots+\mathbf{d}_{r}\right)}=(0)
$$

for all $i=1,2, \ldots, t$. Let $\mathbf{l}_{i}:=\mathbf{k}_{i}+\ell\left(\mathbf{d}_{1}+\mathbf{d}_{2}+\cdots+\mathbf{d}_{r}\right) \in \delta+\Gamma$. Then $\left[R m_{i}\right]_{\mathbf{n}}=(0)$ for all $\mathbf{n} \in C_{\mathbf{l}_{i}} \cap(\delta+\Gamma)$. Hence, by taking a vector $\mathbf{k}=\mathbf{k}(\delta) \in \mathbb{N}^{r}$ such that

$$
C_{\mathbf{k}} \subseteq C_{\mathbf{l}_{1}} \cap C_{\mathbf{l}_{2}} \cap \cdots \cap C_{\mathbf{l}_{t}}
$$

we have that $M_{\mathbf{n}}=\left[M^{(\delta+\Gamma)}\right]_{\mathbf{n}}=(0)$ for all $\mathbf{n} \in C_{\mathbf{k}} \cap(\delta+\Gamma)$. This completes the proof of the claim.

Now, let $\mathbf{k} \in \mathbb{N}^{r}$ be a vector such that

$$
C_{\mathbf{k}} \subseteq C_{\mathbf{k}_{0}} \cap\left[\bigcap_{\delta \in \Delta_{\mathbf{k}_{0}}} C_{\mathbf{k}(\delta)}\right],
$$


where $\mathbf{k}(\delta)$ is the vector in the claim. Then $M_{\mathbf{n}}=(0)$ for all $\mathbf{n} \in C_{\mathbf{k}}$. Indeed, since $\mathbf{n} \in C_{\mathbf{k}_{0}}$, there is a unique expression $\mathbf{n}=\delta+\mathbf{m}$ for some $\delta \in \Delta_{\mathbf{k}_{0}}$ and $\mathbf{m} \in \Gamma$. Hence $\mathbf{n} \in C_{\mathbf{k}(\delta)} \cap(\delta+\Gamma)$ so that $M_{\mathbf{n}}=(0)$ by the claim.

We prove the other implication. Suppose that there exists $\mathbf{k} \in \mathbb{N}^{r}$ such that $M_{\mathbf{n}}=(0)$ for all $\mathbf{n} \in C_{\mathbf{k}}$. We write $M=R m_{1}+R m_{2}+\cdots+R m_{t}$, where $m_{i} \in M_{\mathbf{k}_{i}}$. Then it is enough to show that for any $i=1,2, \ldots, t$, there exists an integer $\ell \geq 0$ such that $R_{++}^{\ell} \cdot m_{i}=(0)$. For the vectors $\mathbf{k}, \mathbf{k}_{i} \in \mathbb{N}^{r}$, there exists an integer $\ell_{0} \geq 0$ such that

$$
\mathbf{k}_{i}+\ell\left(\mathbf{d}_{1}+\mathbf{d}_{2}+\cdots+\mathbf{d}_{r}\right) \in C_{\mathbf{k}} \text { for all } \ell \geq \ell_{0}
$$

by Lemma 2.1. Thus

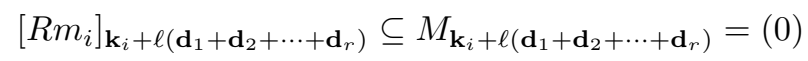

and hence $R_{++}^{\ell} \cdot m_{i}=(0)$.

Lemma 2.3. Let $M=\bigoplus_{\mathbf{n} \in \mathbb{Z}^{r}} M_{\mathbf{n}}$ be a finitely generated $\mathbb{Z}^{r}$-graded $R$-module and let $\mathbf{k}_{0} \in \mathbb{N}^{r}$ and $\delta \in \Delta_{\mathbf{k}_{0}}$. Then there exists a vector $\mathbf{k} \in \mathbb{N}^{r}$ such that

$$
M_{\mathbf{n}} \neq(0) \text { for all } \mathbf{n} \in C_{\mathbf{k}} \cap(\delta+\Gamma)
$$

if $\operatorname{Supp}_{++} M^{(\delta+\Gamma)} \neq \emptyset$.

Proof. Suppose $\operatorname{Supp}_{++} M^{(\delta+\Gamma)} \neq \emptyset$. Assume the contrary, so that for any vector $\mathbf{k} \in \mathbb{N}^{r}$, there exists $\mathbf{n} \in C_{\mathbf{k}} \cap(\delta+\Gamma)$ such that $M_{\mathbf{n}}=(0)$. Write $M^{(\delta+\Gamma)}=$ $R m_{1}+R m_{2}+\cdots+R m_{t}$, where $m_{i} \in M_{\mathbf{k}_{i}}$ and $\mathbf{k}_{i} \in \delta+\Gamma$. Then, for each $\mathbf{k}_{i}$, there exists $\mathbf{n}_{i} \in C_{\mathbf{k}_{i}} \cap(\delta+\Gamma)$ such that $M_{\mathbf{n}_{i}}=(0)$ by the assumption. Therefore $\left[R m_{i}\right]_{\mathbf{n}_{i}}=(0)$ and hence $\left[R m_{i}\right]_{\mathbf{n}}=(0)$ for all $\mathbf{n} \in C_{\mathbf{n}_{i}}$. By taking a cone $C_{\mathbf{k}}$ such that

$$
C_{\mathbf{k}} \subseteq C_{\mathbf{n}_{1}} \cap C_{\mathbf{n}_{2}} \cap \cdots \cap C_{\mathbf{n}_{t}},
$$

we have $\left[M^{(\delta+\Gamma)}\right]_{\mathbf{n}}=(0)$ for all $\mathbf{n} \in C_{\mathbf{k}}$, which implies that $\operatorname{Supp}_{++} M^{(\delta+\Gamma)}=\emptyset$ by Lemma 2.2. This is a contradiction.

\section{Proof of Theorem 1.1}

We are now ready to prove Theorem 1.1. Recall that $R$ is a Noetherian $\mathbb{N}^{r}$-graded ring generated in degrees $\mathbf{d}_{1}, \mathbf{d}_{2}, \ldots, \mathbf{d}_{r}$, where $\mathbf{d}_{1}, \mathbf{d}_{2}, \ldots, \mathbf{d}_{r} \in \mathbb{N}^{r}$ are linearly independent vectors over $\mathbb{R}$, with $R_{\mathbf{0}}=A$. Let $M=\bigoplus_{\mathbf{n} \in \mathbb{Z}^{r}} M_{\mathbf{n}}$ be a finitely generated $\mathbb{Z}^{r}$-graded $R$-module and let $\mathfrak{a}$ be an ideal in $A$.

Proof of Theorem 1.1. Let $\mathfrak{a}=\left(a_{1}, a_{2}, \ldots, a_{p}\right) A$ and let $\mathbf{k}_{0} \in \mathbb{N}^{r}$ be any fixed vector. Then we claim the following:

Claim. For any $\delta \in \Delta_{\mathbf{k}_{0}}$, there exist a vector $\mathbf{k}=\mathbf{k}(\delta) \in \mathbb{N}^{r}$ and a constant $c=c(\delta) \in \mathbb{N} \cup\{\infty\}$ such that grade $\left(\mathfrak{a}, M_{\mathbf{n}}\right)=c$ for all $\mathbf{n} \in C_{\mathbf{k}} \cap(\delta+\Gamma)$.

Let $\delta \in \Delta_{\mathbf{k}_{0}}$ and $L:=M / \mathfrak{a} M$. If $\operatorname{Supp}_{++} L^{(\delta+\Gamma)}=\emptyset$, then there exists $\mathbf{k} \in \mathbb{N}^{r}$ such that $L_{\mathbf{n}}=(0)$ for all $\mathbf{n} \in C_{\mathbf{k}} \cap(\delta+\Gamma)$ by Lemma 2.2. Therefore $M_{\mathbf{n}}=\mathfrak{a} M_{\mathbf{n}}$ so that $\operatorname{grade}\left(\mathfrak{a}, M_{\mathbf{n}}\right)=\infty$ for all $\mathbf{n} \in C_{\mathbf{k}} \cap(\delta+\Gamma)$. Suppose $\operatorname{Supp}_{++} L^{(\delta+\Gamma)} \neq \emptyset$. By Lemma 2.3, there exists $\mathbf{k}_{1} \in \mathbb{N}^{r}$ such that for any $\mathbf{n} \in C_{\mathbf{k}_{1}} \cap(\delta+\Gamma), L_{\mathbf{n}} \neq(0)$ so that $M_{\mathbf{n}} \neq \mathfrak{a} M_{\mathbf{n}}$. Thus, by the grade sensitivity of the Koszul complex, we have that for any $\mathbf{n} \in C_{\mathbf{k}_{1}} \cap(\delta+\Gamma)$,

$$
\operatorname{grade}\left(\mathfrak{a}, M_{\mathbf{n}}\right)=p-\max \left\{i \mid H_{i}\left(\underline{a} ; M_{\mathbf{n}}\right) \neq(0)\right\},
$$


where $H_{i}(\underline{a} ; *)$ denotes the $i$-th Koszul homology module of $*$ with respect to the sequence $\underline{a}=a_{1}, a_{2}, \ldots, a_{p}$. Let

$$
q:=\max \left\{i \mid \operatorname{Supp}_{++}\left(H_{i}\left(\underline{a} ; M^{(\delta+\Gamma)}\right)\right) \neq \emptyset\right\} .
$$

For any $i>q$, since $\operatorname{Supp}_{++}\left(H_{i}\left(\underline{a} ; M^{(\delta+\Gamma)}\right)\right)=\emptyset$, there exists $\mathbf{l}_{i} \in \mathbb{N}^{r}$ such that

$$
H_{i}\left(\underline{a} ; M_{\mathbf{n}}\right)=(0) \text { for all } \mathbf{n} \in C_{\mathbf{l}_{i}} \cap(\delta+\Gamma)
$$

by Lemma 2.2, On the other hand, since $\operatorname{Supp}_{++}\left(H_{q}\left(\underline{a} ; M^{(\delta+\Gamma)}\right)\right) \neq \emptyset$, there exists $\mathbf{l}_{q} \in \mathbb{N}^{r}$ such that

$$
H_{q}\left(\underline{a} ; M_{\mathbf{n}}\right) \neq(0) \text { for all } \mathbf{n} \in C_{\mathbf{l}_{q}} \cap(\delta+\Gamma)
$$

by Lemma 2.3 . Thus, by taking a cone $C_{\mathbf{k}_{2}}$ such that

$$
C_{\mathbf{k}_{2}} \subseteq C_{\mathbf{l}_{q}} \cap C_{\mathbf{l}_{q+1}} \cap \cdots \cap C_{\mathbf{l}_{p}},
$$

we have that for all $\mathbf{n} \in C_{\mathbf{k}_{2}} \cap(\delta+\Gamma)$,

$$
H_{q}\left(\underline{a} ; M_{\mathbf{n}}\right) \neq(0) \text {, and } H_{i}\left(\underline{a} ; M_{\mathbf{n}}\right)=(0) \text { if } i>q .
$$

By taking a cone $C_{\mathbf{k}}$ such that $C_{\mathbf{k}} \subseteq C_{\mathbf{k}_{1}} \cap C_{\mathbf{k}_{2}}$, we get that

$$
\operatorname{grade}\left(\mathfrak{a}, M_{\mathbf{n}}\right)=p-q \text { for all } \mathbf{n} \in C_{\mathbf{k}} \cap(\delta+\Gamma) \text {. }
$$

This completes the proof of the claim.

Now let us take a cone $C_{\mathbf{k}}$ such that

$$
C_{\mathbf{k}} \subseteq C_{\mathbf{k}_{0}} \cap\left[\bigcap_{\delta \in \Delta_{\mathbf{k}_{0}}} C_{\mathbf{k}(\delta)}\right],
$$

where $\mathbf{k}(\delta)$ is the vector in the claim. Then we have the equality

$$
\operatorname{grade}\left(\mathfrak{a}, M_{\mathbf{n}}\right)=\operatorname{grade}\left(\mathfrak{a}, M_{\mathbf{n}+\mathbf{m}}\right)
$$

for all $\mathbf{n} \in C_{\mathbf{k}}$ and all $\mathbf{m} \in \Gamma$. Indeed, since $\mathbf{n} \in C_{\mathbf{k}_{0}}$, there is a unique $\delta \in \Delta_{\mathbf{k}_{0}}$ such that $\mathbf{n} \in \delta+\Gamma$. Thus $\mathbf{n}, \mathbf{n}+\mathbf{m} \in C_{\mathbf{k}(\delta)} \cap(\delta+\Gamma)$ and hence grade $\left(\mathfrak{a}, M_{\mathbf{n}}\right)=$ $\operatorname{grade}\left(\mathfrak{a}, M_{\mathbf{n}+\mathbf{m}}\right)$ by the claim.

If we take each $\mathbf{d}_{i}=(0, \ldots, 0,1,0, \ldots, 0)$ to be the $i$-th standard basis element of $\mathbb{N}^{r}$, then we can readily get the known results in the standard graded cases [6, 7, 8, Moreover, as a direct consequence, we have the stability of the grade in the special nonstandard graded cases considered in [8].

Corollary 3.1. Let $R, M, \mathfrak{a}$ be the same as in Theorem 1.1. Assume that each vector $\mathbf{d}_{i}$ has the form $\mathbf{d}_{i}=(*, \ldots, *, 1,0, \ldots, 0)$, where 1 is in the $i$-th column. Then there exists a vector $\mathbf{k} \in \mathbb{N}^{r}$ such that grade $\left(\mathfrak{a}, M_{\mathbf{n}}\right)$ is constant in the cone $C_{\mathbf{k}}$. Namely, the equality

$$
\operatorname{grade}\left(\mathfrak{a}, M_{\mathbf{n}}\right)=\operatorname{grade}\left(\mathfrak{a}, M_{\mathbf{k}}\right)
$$

holds true for all $\mathbf{n} \in C_{\mathbf{k}}$.

Proof. Let $\mathbf{k}_{0} \in \mathbb{N}^{r}$ be any fixed vector. Then $\Delta_{\mathbf{k}_{0}}=\left\{\mathbf{k}_{0}\right\}$ because of the form of the vectors $\mathbf{d}_{i}$. Thus we have the assertion as a direct consequence of Theorem 1.1.

Colomé-Nin and Elias studied in [4] the asymptotic behavior of $\operatorname{depth}_{A} M_{\mathbf{n}}$ in the case where each $\mathbf{d}_{i}$ has the form $\left(*, \ldots, *, \lambda_{i}, 0, \ldots, 0\right)$ with $\lambda_{i} \neq 0$. This case is also a special case of Theorem 1.1 . 


\section{REFERENCES}

[1] A. L. Branco Correia, S. Zarzuela, On the asymptotic properties of the Rees powers of a module, J. Pure Appl. Algebra 207 (2006) 373-385. MR.2254891 (2007f:13006)

[2] M. Brodmann, Asymptotic stability of $\operatorname{Ass}\left(M / I^{n} M\right)$, Proc. Amer. Math. Soc. 74 (1979) 16-18. MR.521865 (80c:13012)

[3] M. Brodmann, The asymptotic nature of analytic spreads, Math. Proc. Cambridge Philos. Soc. 86 (1979) 35-39. MR.530808 (81e:13003)

[4] G. Colomé-Nin, J. Elias, On the asymptotic depth of multigraded modules, to appear in Comm. Algebra.

[5] F. Hayasaka, Asymptotic stability of primes associated to homogeneous components of multigraded modules, J. Algebra 306 (2006) 535-543. MR2271351 (2008b:13004)

[6] J. Herzog, T. Hibi, The depth of powers of an ideal, J. Algebra 291 (2005) 534-550. MR.2163482 (2006h:13023)

[7] S. McAdam, P. Eakin, The asymptotic Ass, J. Algebra 61 (1979) 71-81. MR554852 (81f:13001)

[8] E. West, Primes associated to multigraded modules, J. Algebra 271 (2004) 427-453. MR2024817(2004m:13004)

Department of Liberal Arts and Sciences, Kagoshima National College of TechNOLOGY, 1460-1 Shinko, HAYATO-ChO, Kirishima-Shi, Kagoshima 899-5193, JAPAN

E-mail address: hayasaka@kagoshima-ct.ac.jp 